\title{
Betatron Phase Advanced Measurement System for the Storage Ring of SRRC
}

\author{
K. H. Hu, Jenny Chen, C. H. Kuo, Demi Lee, K. T. Hsu, K. K. Lin \\ Synchrotron Radiation Research Center, Hsinchu, Taiwan, R. O. C.
}

\begin{abstract}
An extension of existing multiplex BPM electronics which provides the capability for phase advance measurement is implemented. Resonantly excite the stored beam then detect relative betatron phase of all BPMs by lock-in amplifier. Design considerations of the system and preliminary test results are presented in this report. Based on the rapid measurement capability and analysis methodology, possible applications of the system are betatron phase measurement, betatron functions measurement, local coupling parameter study, and determines the errors of the lattice.
\end{abstract}

\section{INTRODUCTION}

Measured betatron function and phase advance information is essential for precision beam-based machine modelling and helpful to optimise performance. Resonance excitations of the stored beam and measure the betatron phase are adopted by many experiments, for example, LEP [1] and CESR [2,3]. The BPM electronics of SRRC is a multiplexing system for precision closedorbit measurement [4]. Using all BPMs for machine optics measurement is highly desirable. A simple functional extension of the SRRC BPM system was implemented recently. A log-amplifier video detector mezzanine is implemented and installed in all BPMs electronic. Using lock-in amplifier to detect coherent oscillation with resonance excitation can support fast betatron phase measurement. Application of the system includes betatron phase measurement, betatron functions measurement, local coupling parameter measurement, and determines the errors of the lattice.

\section{MEZZANINE MODULE}

The multiplexing BPM electronics is a commercial unit (Bergoz's MX-BPM). It is designed for averaged beam position measurement with micron resolution. The electronics is composed of low pass filter, GaAs RF switch, band pass filter, high performance mixer and IF amplifier, quasi-synchronous detector, analog demultiplexer and position computation circuitry. To observe betatron oscillation, wide bandwidth detector is needed. The IF bandwidth of MX-BPM before quasisynchronous detector is larger than $5 \mathrm{MHz}$ which is sufficient for betatron oscillation observation. This simple log amplifier detector was implemented due to its simplicity, no need of gain control, and small component counts. This mezzanine supports larger than $50 \mathrm{~dB}$ dynamic range. When mezzanine module is engaged, AGC function of MX-BPM is disabled and single button is enable. The mezzanine is installed near the IF amplifier and detector circuitry. Small signal sensitivity is limited to about $-60 \mathrm{dBm}$ that is due to deteriorate of the operation of PLL in synchronous detector. The functional block diagram of the mezzanine is shown in Figure 1. The mezzanine demodulates bunches signal at IF frequency (21.4 MHz). The reconstructed position error due to log conformance is acceptable for small oscillation amplitude.

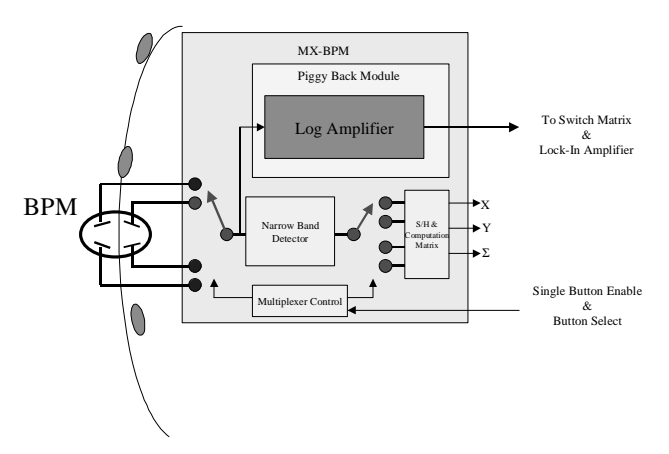

Figure 1: Functional block diagram of BPM processing electronics.

\section{BETATRON PHASE MEASUREMENT}

Resonance excitation is done by a dedicated phase locked loop. The loop includes functions of search and tracking. Coherent signal is detected by a commercial logratio BPM electronics (Bergoz's LR-BPM) [5]. MX-BPM mezzanine circuit detects betatron oscillation at every BPM button. A RF lock-in amplifier is used to measure the amplitude and phase of the coherent oscillation. Basic concept of the operation is shown in Figure 2. Each MXBPM which is installed a log video detector achieves wide bandwidth output. A RF multiplexer and a select circuitry are used to select single button signal. Measured revolution harmonic phase shift compensates the cable and detector circuitry delay. Since the betatron oscillation of the storage ring is between $300 \mathrm{kHz}$ and $800 \mathrm{kHz}$ and is out of the working frequency range of a low frequency lock-in amplifier, a RF lock-in amplifier is required. The lock -in amplifier detects the relative phase of the excitation signal and coherent beam signal at each BPM button.

The phase $\phi_{i}$ of the beam signal at the ith detector relative to the excitation reference signal gives the betatron phase hi at the BPM: 


$$
\phi(i)=\theta(i)+2 \pi m_{i}+\theta_{a}-----------(1)
$$

Where $m i$ is an integer to correct for the factor that phases are always measured modulo $2 \pi$, and $\theta \mathrm{a}$ is a constant independent of the BPM being used that accounts for phase shifts in any filters, etc.

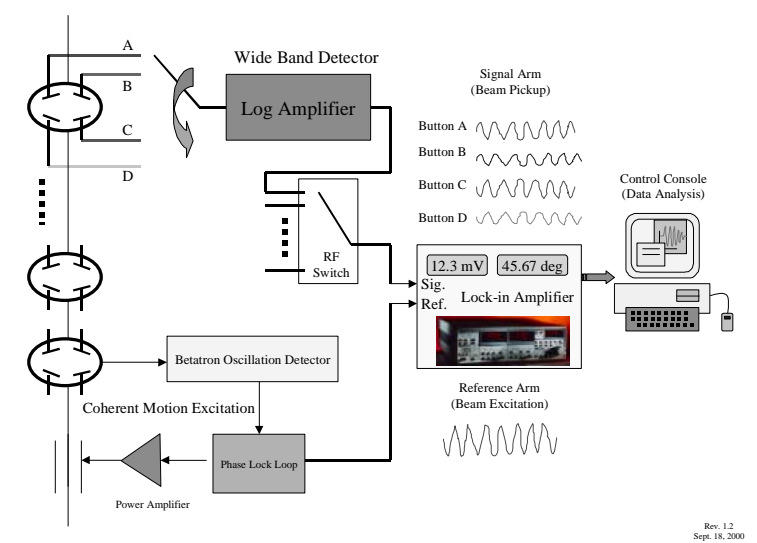

Figure 2: Concepts of betatron phase and local coupling measurement.

The coherent beam signal is transmitted and processed by different electronics. To compensate different cable delay and phase shift of the electronics are based on the fact that all detector signals exhibit a strong component at revolution frequency. The phase of this component varies by $\Delta$ two independent of any betatron oscillations. Measuring the phase of the signal component at wo for each detector thus determines the value of $\Delta \mathrm{T}$.

The implementations are shows in Figure 3. The system consists of control console, network, resonance excitation system, digital I/O module, MX-BPM (include Log video detector mezzanine), analog switch module, lock-in amplifier and software supports to assistant data acquisition and analysis. The operation of the system is controlled by Matlab scripts. Develops Matlab scripts to support data acquisition and analysis work is on going.

An experimental procedure for betatron phase advance is to excite coherent betatron oscillation firstly. Select signal form specific button of selected BPM firstly. Acquire phase and amplitude data by lock-in amplifier is the second step. Repeating the procedure until all button data are acquired. Performed analysis is the last step.

Each cable of connects BPM button and MX-BPM electronic processing module that cable length is difference, cable length difference can cause error of phase advance measurement system. Using results of calibration to correct the raw data. This procedure is essential to eliminate the difference of phase shift of different electronics and cables.

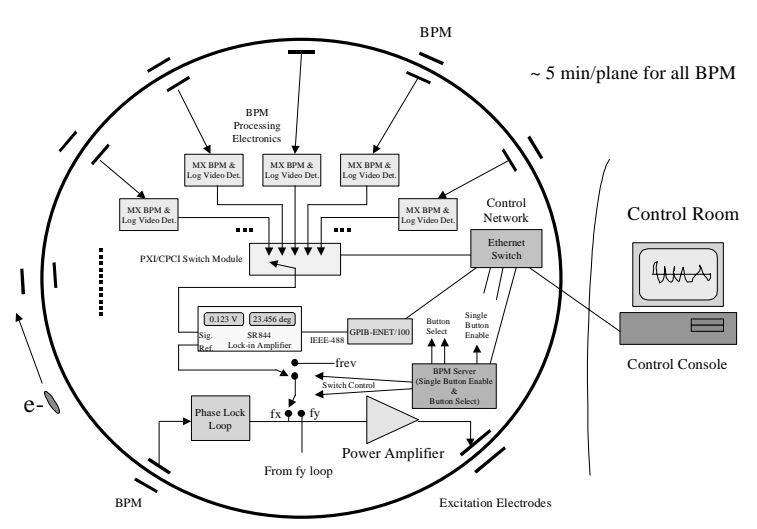

Figure 3: Block diagram of the experimental setup.

\section{PRELIMINARY BEAM TEST}

System integration of the measurement system was done recently. All MX-BPM are installed with log video mezzanines. Preliminary beam test shown that the basic operation principle is working properly. Reproducibility of the measurement is good qualitatively at this moment. Quantitative analysis of the system performance is under investigated. In figure 4 shown that resonance oscillation of the store beam. Upper trace is the output of log ratio BPM. Lower trace is the output of wide band piggyback module installed on MX-BPM. It can observe betatron oscillation of stored beam clearly. The output of LR-BPM shown a vertical oscillation with amplitude about $1 \mathrm{~mm}$. The output of MX-BPM wide band, that output amplitude variation about $10 \mathrm{mV}$. The signal strength is enough for lock-in amplifier provide a reliable reading.

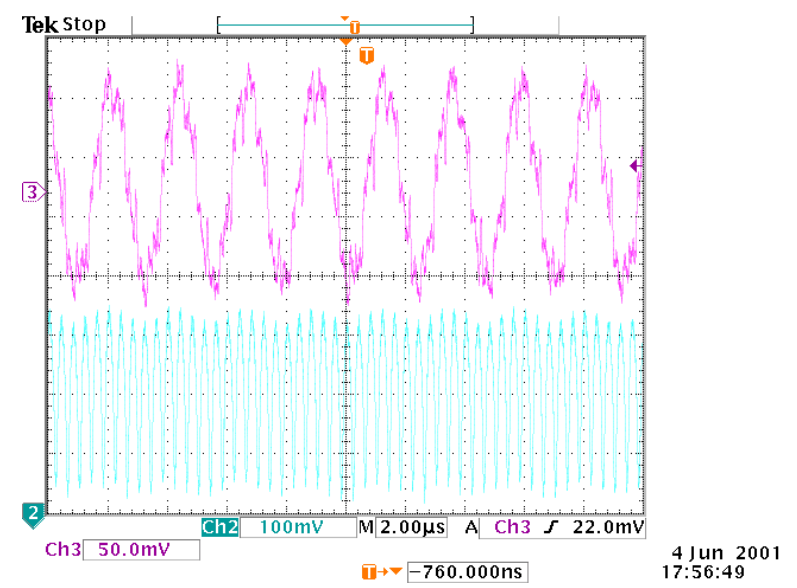

Figure 4: Resonance excitations of the stored beam, to observe performance of piggyback module and LR-BPM.

Phase advance of the storage ring shown in Figure 5. In figure (5a) is phase advance of horizontal plane; (5b) is phase advance of vertical plane.

Measured phase advance compared with model calculation is shown in Figure 6. There are discrepancies in data. A systematic study will be started soon. 


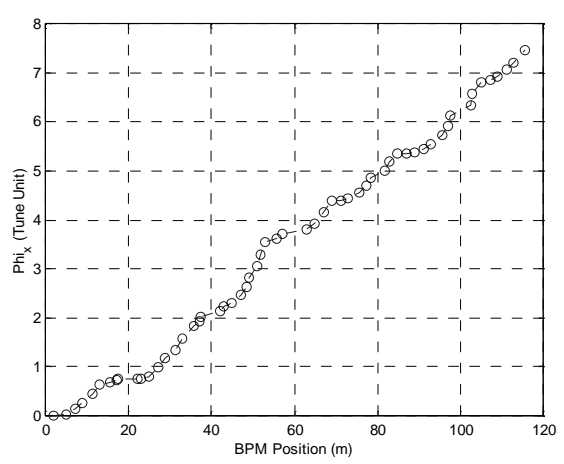

(a) Horizontal

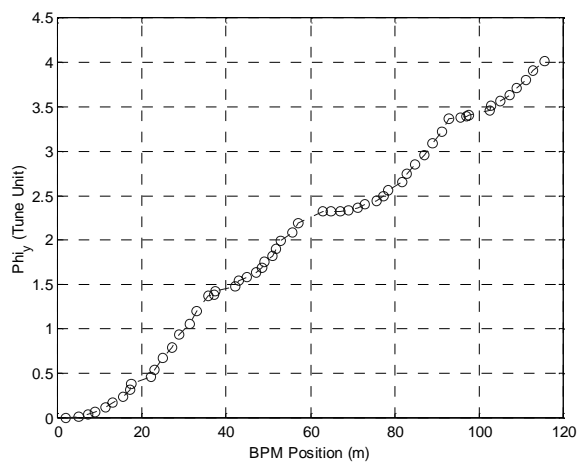

(b) Vertical

Figure 5: Measured phase advance of the storage ring.

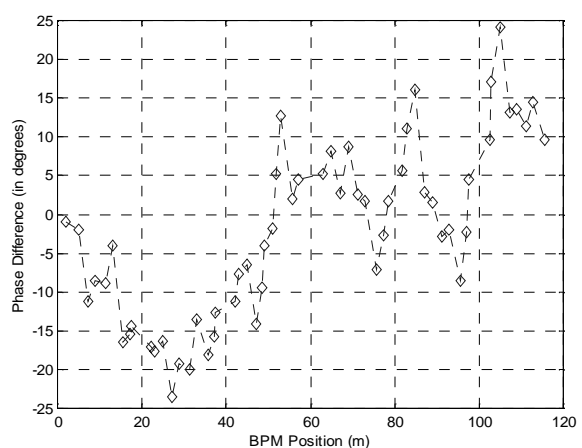

(a) Horizontal

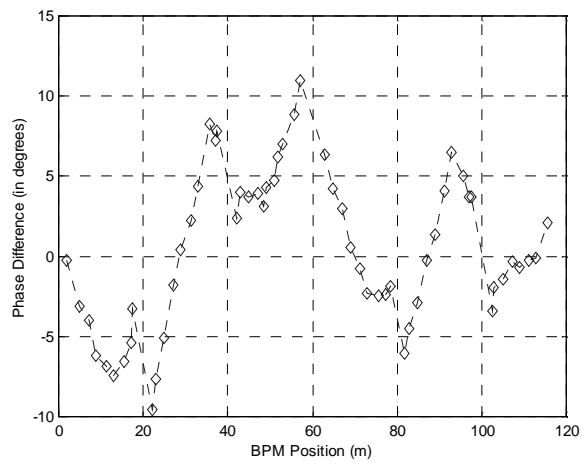

(c) Vertical.

Figure 6: Difference between measured phase advance and model phase advance of the storage ring.

\section{FUTURE WORKS AND PLANS}

Performance of the system should be investigated further and improve to acquire a reliability phase data. The betatron phase advances are measured at all BPM sites quickly. The measured betatron phase can be used to extract betatron function by the relationship of $1 / \beta_{x, y}=$ $\mathrm{d} \phi_{\mathrm{x}, \mathrm{y}} / \mathrm{ds}$. The horizontal and vertical components of the oscillations at a BPM can be resolved allowing for extraction of the local coupling parameters by analysis using parameterised $\overline{\mathrm{C}}$ matrix [3] coupling analysis. Analysis tools to support the analysis is also a Matlab scripts which is under development.

\section{ACKNOWLEDGEMENT}

The authors appreciate brainstorming discussion with Jim Hinkson and Julien Bergoz. David Sagan's explanation of the ideas of betatron phase measurement is beneficial for this works. Help of the operation group are also highly appreciated.

\section{REFERENCES}

[1] P. Castro, J. Borer, A. Burns, G. Morpurgo, and R. Schmidt, Pac93, p.2103 (1993).

[2] P. Bagley and D. Rubin, "Correction of Transverse Coupling in a Storage Ring", PAC89, p. 874 (1989).

[3] D. Sagan, R. Meller, R. Littauer, and D. Rubin, "Betatron phase and coupling measurements at the Cornell Electron/Positron Storage Ring", Published 5 Sept. 2000, E092801 Phys. Rev. ST Accel. Beams and reference therein.

[4] Beam Position Monitor User's Manual, Rev. 1.5.3, BERGOZ Instrumentation.

[5] Log-ratio Beam Position Monitor User's Manual, Rev. 0.1, BERGOZ Instrumentation. 